\title{
Cardiovascular responses to a glucose drink in young male individuals with overweight/obesity and mild alterations in glucose metabolism, but without impaired glucose tolerance
}

\author{
Cathriona R. Monnard ${ }^{1}$. Erik Konrad Grasser ${ }^{1}$ (D)
}

\begin{abstract}
Purpose Little is known about whether mild aberrations in glucose metabolism, which are seen in overweight/obese subjects (OW/OB) without impaired glucose tolerance, affect regulator control elements for blood pressure homeostasis. Methods Hence, we measured in age-matched male subjects with normal weight $\left(n=16\right.$; BMI $\left.=22.4 \mathrm{~kg} \mathrm{~m}^{-2}\right)$ and OW/OB $(n=$ $11 ; \mathrm{BMI}=28.6 \mathrm{~kg} \mathrm{~m}^{-2}$ ) continuous beat-to-beat blood pressure, heart rate, stroke volume, myocardial contractility and baroreflex sensitivity during a $30 \mathrm{~min}$ baseline and for $120 \mathrm{~min}$ after the ingestion of $75 \mathrm{~g}$ glucose dissolved in $300 \mathrm{~mL}$ tap water (OGTT). Blood samples for the assessment of plasma glucose and insulin were collected at baseline and every $30 \mathrm{~min}$ after the drink and homeostatic model assessment of insulin resistance (HOMA-IR) was calculated.

Results At baseline, glucose $\left(5.3 \pm 0.4 \mathrm{SD}\right.$ vs $5.0 \pm^{-1} ;$ p.4-10101), linsulin $\left(7.4 \pm 0.4 \mathrm{vs} 3.7 \pm 2.7 \mathrm{mU} \mathrm{L} \quad{ }^{-1} ; p=\right.$ $0.02)$ and HOMA-IR $(1.8 \pm 1.3$ vs $0.8 \pm 0.6 ; p=0.01)$ were significantly higher in subjects with OW/OB, but none classified as having impaired glucose tolerance (plasma glucose levels $<7.8 \mathrm{mmol} \mathrm{L}^{-1}$ at 120 min post-OGTT) or hypertension (all $<130 / 80 \mathrm{mmHg}$ at baseline). In response to the glucose drink, and in comparison to subjects with normal weight, we observed in subjects with OW/OB a trend towards increased plasma insulin levels $(+7445 \pm 4858$ vs. $\left.+4968 \pm 1924 \mathrm{mU} \mathrm{h} \mathrm{L}^{-1} ; p=0.08\right)$, which was not seen for blood glucose $(p=0.59)$. Moreover, subjects with OW/OB showed impaired peripheral vaso-dilation, diminished heart rate and myocardial contractility responses but increased peripheral pulse pressure (all $p<0.05$ ). Conclusions Young male subjects with $\mathrm{OW} / \mathrm{OB}$, but without glucose intolerance or hypertension, showed attenuated periph-eral vasodilation and diminished cardiac responses to a glucose drink.
\end{abstract}

Keywords Sugar $\cdot$ Insulin resistance $\cdot$ Obesity $\cdot$ Blood pressure

\section{Introduction}

Currently, cardiovascular diseases are the world's leading cause of morbidity and mortality [1]. Although high blood pressure is the largest contributor to cardiovascular diseaserelated mortality (40\% of all deaths) [2], overweight and obesity are also significant modifiable risk factors [3, 4]. On one hand, people with overweight and obesity often exhibit signs of impaired glucose metabolism, which can increase the risk of type 2 diabetes mellitus [5], whilst

Erik Konrad Grasser

erikkonrad.grasser@unifr.ch

1 Department of Endocrinology, Metabolism and Cardiovascular System, Faculty of Science and Medicine, University of Fribourg, Chemin du Musée 5, 1700 Fribourg, Switzerland impaired glucose tolerance and type 2 diabetes mellitus are both major risk factors for cardiovascular diseases [6]. On the other hand, central abdominal obesity (i.e. the excess accumulation of body fat around visceral organs and in close proximity to the liver) might be a powerful candidate for increasing the risk of obesity-related hypertension [7].

In this context, it has recently been suggested that impaired glucose tolerance may adversely affect the cardiovascular system by impairing glycaemic-induced blood pressure regulation [8]. According to this postulation, alterations in glucose metabolism are characterized by elevated levels of insulin and raised sympathetic neural activity at rest [8, 9], an additional surge in insulin secretion following glucose ingestion might then cause further stimulation in sympathetic nerve activity with a potential impact on total peripheral resistance $[8,10]$. This, in turn, may lead to diminished peripheral vasodilation that could adversely affect blood 
pressure regulation $[8,10]$. However, and to the best of our knowledge, it is currently not known whether early stage aberrations in glucose metabolism, i.e. mild increases in fasting glucose and insulin blood levels, but before impaired glucose tolerance emerges, might adversely affect regulatory control elements for blood pressure homeostasis.

We, therefore, investigated in young male Caucasian subjects with overweight/obesity (body mass index between 25.0 and $34.9 \mathrm{~kg} \mathrm{~m}^{-2}$ ) and age-matched Caucasian subjects with normal weight (body mass index between 18.5 and $24.9 \mathrm{~kg} \mathrm{~m}^{-2}$ ) hemodynamic and metabolic responses to a glucose drink. We hypothesized that subjects with overweight/obesity, but without impaired glucose tolerance, display altered glucose metabolism in response to a glucose drink, which consequently affects hemodynamic regulation, characterized by a diminished vasodilatory effect of insulin on peripheral resistance vessels with accompanying cardiac responses.

\section{Methods}

\section{Subjects}

Taking data from a previously published study from our laboratory [11], we reanalysed data from 24 male Caucasian subjects (six were removed due to impaired glucose tolerance test) and added data from 3 unpublished obese male Caucasian subjects. Hence, we included 27 subjects in our final analysis for the current paper. Subjects were students (and their friends) recruited from the university surroundings. Eligibility criteria included: Caucasian, male, healthy, non-smoker, 165-200 cm in height, 20-45 years old with a body mass index ranging from 18.5 to $34.9 \mathrm{~kg} \mathrm{~m}^{-2}$. Exclusion criteria were as follows: individuals with pre-existing disease (i.e. arterial hypertension defined by blood pressure values above $130 / 80 \mathrm{mmHg}$ [12], type 2 diabetes defined by fasting plasma glucose levels above $7.0 \mathrm{mmol} \mathrm{L}^{-1}$ [13]) or who were taking any medication affecting cardiovascular regulation as well as competition athletes and individuals with a daily exercise workload exceeding 60 min per day. In addition, and after the analysis of the study results, subjects diagnosed with impaired glucose tolerance, based on the following criteria in response to the oral glucose tolerance test, were also excluded: showing either (a) fasting glucose levels $\geq 6.1 \mathrm{mmol} \mathrm{L}^{-1}$ (126 mg dL $\left.{ }^{-1}\right)$ [13], or (b) 2-h glucose $\geq 7.8 \mathrm{mmol} \mathrm{L}^{-1}\left(140 \mathrm{mg} \mathrm{dL}^{-1}\right)$ [13, 14]. This study was conducted according to the guidelines laid down in the Declaration of Helsinki and local ethical committee approval was obtained for all procedures involving human subjects. All subjects provided written consent prior to the start of the study.

\section{Study design}

All experiments took place in a quiet, temperature-controlled $\left(22 \pm 1{ }^{\circ} \mathrm{C}\right)$ laboratory and began at 08.00 a.m. following a 12-h overnight fast. Subjects were requested to avoid alcohol, caffeine and physical activity for $24 \mathrm{~h}$ prior to the experiment, and to use public transportation to reach the laboratory. On arrival, subjects were asked to use the restroom if necessary and then to sit in a custom-made and comfortable chair. Electrocardiography and impedance electrodes were positioned together with upper arm and blood pressure cuffs placed on the fingers according to the manufacturer's recommendations [11]. Then, the upper body was covered with a light blanket and an 18-gauge $1.3 \times 33-\mathrm{mm}$ one-way Teflon catheter was inserted in the left cubital vein. This was followed by a variable period for attainment of cardiovascular stability, which was usually between 20 and $30 \mathrm{~min}$. Then, baseline cardiovascular recordings were performed over a $30 \mathrm{~min}$ period, which was immediately followed by a baseline blood sample. The ingestion of the glucose drink $[75 \mathrm{~g}$ of anhydrous D-glucose, dissolved in $300 \mathrm{~mL}$ water at room temperature $\left(22^{\circ} \mathrm{C}\right)$ ], over $4 \mathrm{~min}$, immediately followed the initial blood sample. Cardiovascular recordings then resumed and continued for another $120 \mathrm{~min}$ and blood samples were drawn every $30 \mathrm{~min}$ thereafter for up to $120 \mathrm{~min}$ postdrink. In order to avoid boredom during the study [15], subjects watched calm documentaries on a television placed in front of them.

\section{Cardiovascular measurements}

Cardiovascular and hemodynamic parameters (systolic blood pressure, diastolic blood pressure, RR-interval (RRI), stroke volume, and the Heather index) were measured continuously using beat-to-beat equipment (Task Force Monitor, CNSystems, Medizintechnik, Graz, Austria), which generated data sampled at a rate of $1000 \mathrm{~Hz}$ and stored on a hard disc for later analysis $[11,16]$. Continuous blood pressure monitoring followed the Penaz principle from either the index or middle finger of the right hand and was calibrated to oscillometric brachial blood pressure measurements on the contralateral arm without perturbations caused by the calibration signal [11]. This technique has been validated against invasive and continuous arterial measurements for its precision and accuracy [17] and provided an acceptable agreement during normotensive conditions with interchangeable blood pressure recordings [18]. We used height-adjustable tables for reliable horizontally aligned placement of blood pressure cuffs, i.e., table heights were adjusted to the height of the right atrium (forth-intercostal space), in order to avoid misleading blood pressure readings based on deviations 
from heart level [11]. Moreover, an adaptive cushion was placed on top of each table where the subject's forearms rested comfortably throughout the study [11]. Blood pressure cuff sizes were chosen according to the upper arm circumference [11]. Cardiac stroke volume and the Heather index (as a marker for positive inotropy of the heart) [19] were derived through impedance cardiography measurements. In this context, the Heather index is calculated as $\left(\mathrm{dZ} / \mathrm{dt}_{\max }\right) / \mathrm{Z}_{0}\left(t_{\mathrm{dZmax}}-t_{\mathrm{QRS}}\right)$ and reflects the changes in impedance to the time in which they are performed from the electrical onset to the peak mechanical action [20]. Impedance cardiography, in which changes in thoracic impedance are converted to reflect changes in thoracic fluid content/volume over time, were performed based on the original Kubicek approach [21], but using an improved estimate of thoracic volume [22]. Finally, we determined Baroreflex sensitivity from spontaneous fluctuations in continuously recorded blood pressure and cardiac interval using the sequence technique [16, 23].

\section{Anthropometric and body composition measurements}

Standing height was measured using a mechanical column scale with integrated stadiometer (Seca model 709, Hamburg, Germany) and body weight using an electronic scale (Tanita Corporation, Amsterdam, The Netherlands) [11]. Body mass index was calculated as the ratio of weight $(\mathrm{kg})$ and height squared $\left(\mathrm{m}^{2}\right)$, whilst body composition was measured as previously published by our group [11, 24] using two validate techniques: (a) multi-frequency bioelectrical impedance analyzer (BIA; Inbody 720, Biospace Co., Ltd, Seoul, Korea) for the assessment of total fat mass (kg), fat free mass $(\mathrm{kg})$, and visceral fat mass $\left[\mathrm{cm}^{3}\right][11]$ and (b) a dual-frequency BIA device (ViScan AB-140, Tanita) for the assessment of total trunk (abdominal) fat [\%] [24]. The ViScan technique has been validated against magnetic resonance imaging for the prediction of abdominal fat percentage [25].

\section{Blood sample measurement}

Blood samples were collected and used to measure plasma glucose and insulin as described previously [11]. Briefly, samples were collected in appropriate BD vacutainers (Becton, Dickinson Allschwil, Switzerland) and were processed and centrifuged according to the manufacturer's instructions to separate plasma from whole blood. Aliquots were then stored in $2 \mathrm{ml}$ cryovials and plasma samples for glucose analysis were immediately frozen and stored at $-80{ }^{\circ} \mathrm{C}$, whilst plasma samples for the analysis of insulin were stored at $-20{ }^{\circ} \mathrm{C}$. After thawing the samples, assays were performed according to the manufacturer's instructions: $(i)$
Glucose by the reference method with hexokinase Glucose HK Gen.3 (cobas c 501, Roche Diagnostics) and (ii) insulin by using an ELISA assay kit (Mercodia, Uppsala, Sweden). Phlebotomy and blood sample processing were carried out in accordance with institutional safety requirements for the handling of human biological specimens.

\section{Data collection and processing}

Beat-to-beat values of systolic blood pressure, diastolic blood pressure, heart rate, stroke volume and the Heather index were averaged every 30 min during baseline and every 30 min during the 120 min post-drink period in order to match blood data. Overall changes were calculated as averages over the entire 120 min measurement period with baseline values subtracted [11]. Pulse pressure was calculated as systolic minus diastolic blood pressure and heart rate was calculated from the appropriate RR-interval [11]. Cardiac output was computed as the product of stroke volume and heart rate, whilst total peripheral resistance was calculated as mean blood pressure/cardiac output [11].

Area under curve analysis by the trapezoid method was used to assess glucose-induced responses for glucose and insulin [11]. Homeostatic model assessment for insulin resistance (HOMA-IR) was calculated as [fasting glucose $\left(\mathrm{mmol} \mathrm{L}{ }^{-1}\right) \times$ fasting insulin $\left.\left(\mathrm{mIU} \mathrm{L}^{-1}\right)\right] / 22.5$ [26].

\section{Statistical analysis}

Statistical analyses were performed using Statistix (version 8.0, Analytical Software, St. Paul, MN, USA) and GraphPad Prism (Version 6, GraphPad Software, Inc., La Jolla, USA) and all values are reported as mean \pm standard deviation (SD).

In order to elucidate potential differences between the two groups, we performed statistical analysis with a twofactor mixed-design ANOVA with time as within-subject factor (i.e. baseline, 30, 60, 90 and $120 \mathrm{~min}$ ) and the grouping as between-subject factor (i.e. overweight/obese versus normal weight subjects). Testing for normal distribution was performed using the D'Agostino \& Pearson omnibus normality test and repeated measures ANOVA with Dunnett's multiple comparison post hoc testing (for systolic blood pressure, diastolic blood pressure, pulse pressure, heart rate and stroke volume) or the Friedman test with Dunns post hoc testing (for cardiac output, total peripheral resistance, Heather index, and Baroreflex sensitivity) was used to test for changes over time from baseline levels whenever significant differences were observed from the two-factor mixeddesign ANOVA. All reported $p$-values were two-sided and significance was set at $p<0.05$. 


\section{Results}

\section{Baseline characteristics of the study subjects}

A total of 27 male subjects were included in this study, of which 16 were normal weight and 11 were overweight/ obese. Baseline anthropometric-, blood-, and cardiovascular data prior to consuming the glucose drink are presented in Tables 1 and 2. Based on these data, none of the subjects could be classified as having type 2 diabetes or hypertension, and groups did not differ based on age (Tables 1 and 2).

Compared to subjects with normal weight, subjects with overweight/obesity had significantly greater body mass index, fat mass, fat free mass, visceral fat, trunk (abdominal) fat, glucose, insulin and HOMA-IR (all $p<0.05$, Table 1). With the exception of the Heather index (higher in subjects with normal weight, $p=0.01$ ), as a marker of myocardial contraction force [27-29], and baroreflex sensitivity (higher in subjects with overweight/ obesity, $p=0.03$ ) no other hemodynamic parameter differed between the two groups at baseline (Table 2).

\section{Glucose drink-induced hemodynamic changes}

Figures 1, 2, 3 depict systolic-, diastolic-, and pulse pressure as well as heart rate, total peripheral resistance, the Heather index, cardiac output, stroke volume and baroreflex sensitivity in response to a glucose drink as (i) time course (left panels, respectively) and as (ii) cumulative response equivalent to an area under the curve analysis (right panels, respectively).

Unlike in subjects with normal weight, those with overweight/obesity showed a significant increase in systolic
Table 1 Baseline

anthropometric- and blood data for male subjects with normal weight $(n=16)$ and subjects with overweight/obesity $(n=11)$

Table 2 Baseline cardiovascular parameters for male subjects with normal weight $(n=16)$ and subjects with overweight/ obesity $(n=11)$

\begin{tabular}{|c|c|c|c|c|c|}
\hline Variable & $\begin{array}{l}\text { Normal weight } \\
\text { Mean } \pm \text { SD }\end{array}$ & Range & $\begin{array}{l}\text { Overweight/obese } \\
\text { Mean } \pm \text { SD }\end{array}$ & Range & $p$ value \\
\hline Age, years & $23.7 \pm 3.1$ & $20-30$ & $24.2 \pm 4.4$ & $20-31$ & 0.73 \\
\hline Height, cm & $177 \pm 6$ & $168-191$ & $181 \pm 7$ & $166-189$ & 0.15 \\
\hline Weight, kg & $69 \pm 5$ & $58-80$ & $94 \pm 16$ & $71-119$ & $<0.005$ \\
\hline Waist circumference, $\mathrm{cm}$ & $79 \pm 4$ & $74-87$ & $97 \pm 10$ & $82-114$ & $<0.005$ \\
\hline Body mass index, $\mathrm{kg} \times \mathrm{m}^{-2}$ & $22.2 \pm 1.6$ & $19.2-24.4$ & $28.7 \pm 3.2$ & $25.2-34.2$ & $<0.005$ \\
\hline Body fat mass, $\mathrm{kg}$ & $9.0 \pm 2.7$ & $4.5-15.2$ & $24.4 \pm 9.6$ & $14.1-42.5$ & $<0.005$ \\
\hline Body fat mass, $\%$ & $12.9 \pm 3.4$ & $6.3-18.3$ & $25.0 \pm 5.8$ & $17.8-34.8$ & $<0.005$ \\
\hline Body fat free mass, $\mathrm{kg}$ & $61 \pm 6$ & $49-70$ & $70 \pm 8$ & $57-81$ & $<0.005$ \\
\hline Visceral fat $\mathrm{cm}^{3}$ & $47 \pm 16$ & $21-76$ & $103 \pm 36$ & $44-175$ & $<0.005$ \\
\hline Trunk fat $\%$ & $14.5 \pm 4.2$ & $5.0-22.3$ & $28.5 \pm 6.3$ & $19.6-38.5$ & $<0.005$ \\
\hline Glucose mmol $\times \mathrm{L}^{-1}$ & $5.0 \pm 0.3$ & $4.3-5.4$ & $5.3 \pm 0.4$ & $4.7-5.9$ & 0.01 \\
\hline Insulin $\mathrm{mU} \times \mathrm{L}^{-1}$ & $3.7 \pm 2.7$ & $0.5-10.8$ & $7.4 \pm 4.9$ & $2.6-17.5$ & 0.02 \\
\hline HOMA-IR & $0.8 \pm 0.6$ & $0.1-2.5$ & $1.8 \pm 1.3$ & $0.6 \mathrm{v} 4.6$ & 0.01 \\
\hline
\end{tabular}

$n$ number of subjects, SD Standard deviation, HOMA-IR homeostasis model assessment of insulin resistance, Statistical analysis was performed using an unpaired student's $t$ Test and $p<0.05$ was considered as a significant difference

\begin{tabular}{|c|c|c|c|}
\hline Variable & $\begin{array}{l}\text { Normal weight } \\
\text { Mean } \pm \text { SD }\end{array}$ & $\begin{array}{l}\text { Overweight/obese } \\
\text { Mean } \pm \text { SD }\end{array}$ & $p$ value \\
\hline Systolic blood pressure, $\mathrm{mm} \mathrm{Hg}$ & $114 \pm 7$ & $113 \pm 4$ & 0.40 \\
\hline Diastolic blood pressure, $\mathrm{mm} \mathrm{Hg}$ & $73 \pm 5$ & $71 \pm 5$ & 0.28 \\
\hline Pulse pressure, $\mathrm{mm} \mathrm{Hg}$ & $42 \pm 4$ & $42 \pm 4$ & 0.88 \\
\hline Heart rate, beats $\times \min ^{-1}$ & $66 \pm 7$ & $62 \pm 7$ & 0.17 \\
\hline Stroke volume, $\mathrm{mL}$ & $79 \pm 12$ & $86 \pm 17$ & 0.24 \\
\hline Cardiac output, $\mathrm{L} \times \min ^{-1}$ & $5.2 \pm 0.7$ & $5.2 \pm 0.7$ & 0.84 \\
\hline Total peripheral resistance, $\mathrm{mm} \mathrm{Hg} \times \min \times \mathrm{L}^{-1}$ & $17.1 \pm 2.6$ & $16.5 \pm 2.3$ & 0.55 \\
\hline Heather index, $1 \times \sec ^{-2}$ & $0.24 \pm 0.04$ & $0.20 \pm 0.05$ & 0.01 \\
\hline Baroreflex sensitivity, msec $\times \mathrm{mmHg}^{-1}$ & $17.5 \pm 8.5$ & $26.7 \pm 12.7$ & 0.03 \\
\hline
\end{tabular}

$n$ number of subjects, $S D$ Standard deviation, Statistical analysis was performed using an unpaired students $t$-Test and $p<0.05$ was considered as a significant difference 
Fig. 1 Left panels a-c Time course of changes $(\Delta)$ from baseline values over the following $30 \mathrm{~min}, 30-60 \mathrm{~min}$, 60-90 min and 90-120 min post-drink for systolic blood pressure $(S B P)$, diastolic blood pressure $(D B P)$ and pulse pressure $(P P)$, respectively. \$: Significant interaction effect between subjects with normal weight and subjects with overweight/obesity using two-way mixed model ANOVA analysis. Right panels represent individual mean changes in SBP, DBP and PP over 120 min post-drink, from which baseline values are subtracted. Left panel values are reported as mean \pm standard deviation, whilst right panel data are presented as an individual scatter dot plot with a mean (bold horizontal lines indicate global mean changes derived from averaging all individual mean changes). $* p<0.05$ and $* * * p<0.005$ statistically significant difference when compared to baseline values, respectively
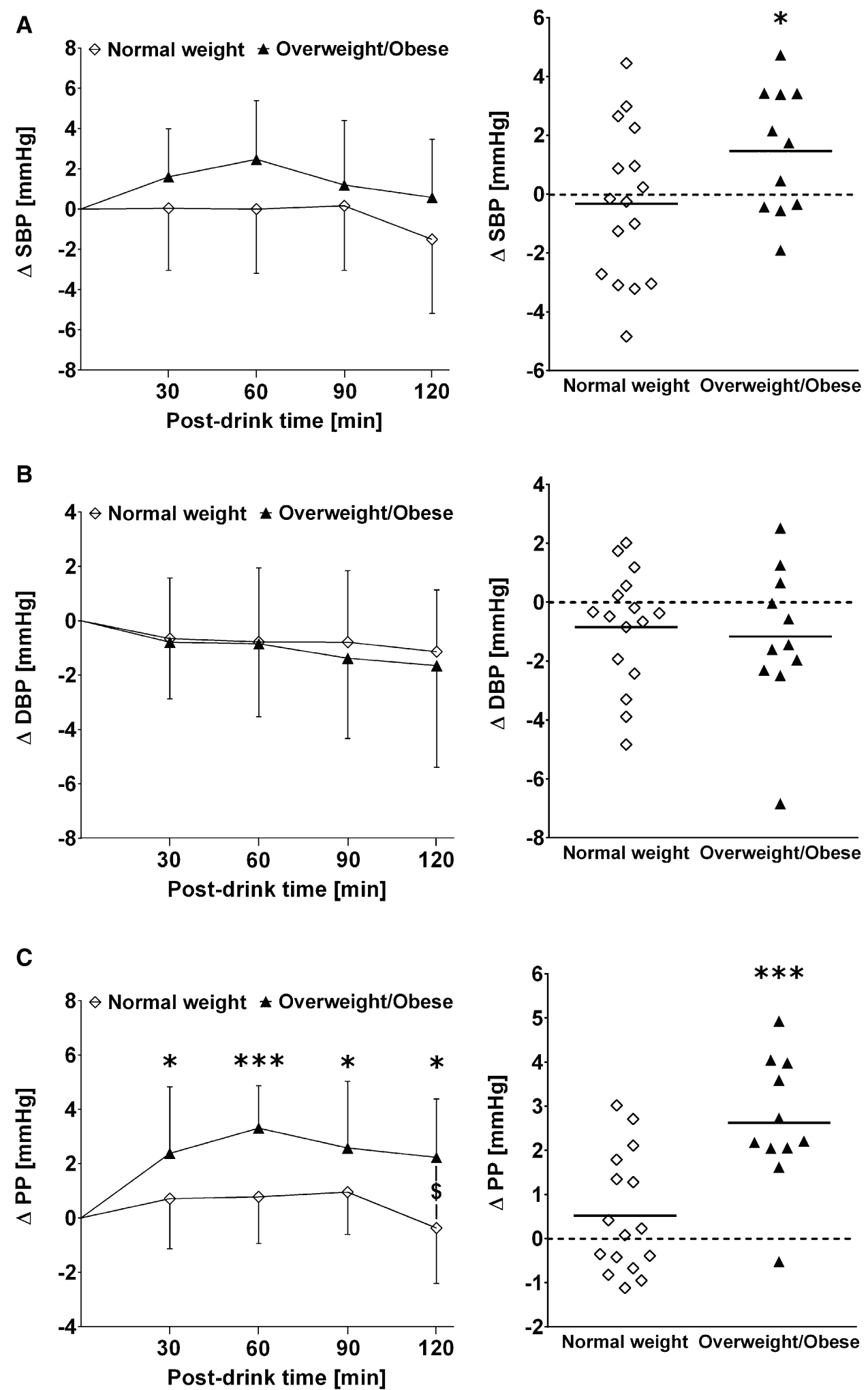

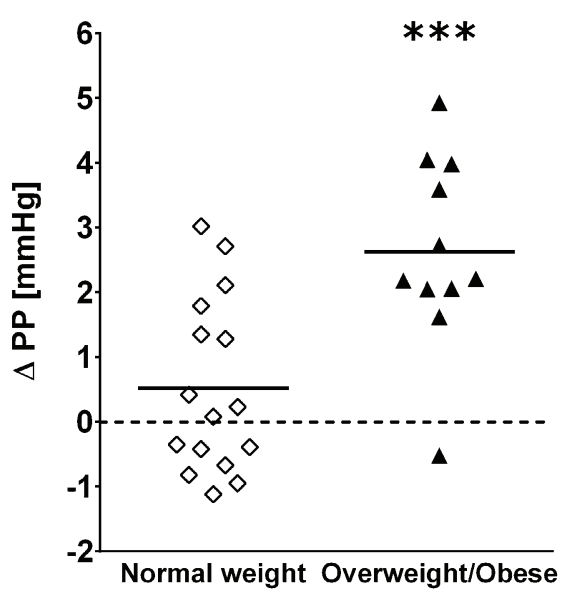

blood pressure $(+1.5 \pm 2.1 \mathrm{mmHg}, p<0.05)$ with unchanged diastolic blood pressure (Fig. 1, a and b, both panels), which lead to a significant increase in postprandial pulse pressure that was different from normal weight subjects $(+2.6 \pm 1.5$ versus $+0.5 \pm 1.4 \mathrm{mmHg}$, two-way mixed model ANOVA interaction effect: $p=0.02$; Fig. 1c, left and right panel).

Heart rate increased in response to the glucose drink in both groups, although to a lesser extent in subjects with 
Fig. 2 Left panels a-c Time course of changes $(\Delta)$ from baseline values over the following $30 \mathrm{~min}, 30-60 \mathrm{~min}$, 60-90 $\mathrm{min}$ and 90-120 min post-drink for heart rate $(H R)$, total peripheral resistance (TPR) and the Heather index $(H I)$, respectively. \$: Significant interaction effect between subjects with normal weight and subjects with overweight/ obesity using two-way mixed model ANOVA analysis. Right panels represent individual mean changes in HR, TPR and HI over 120 min post-drink, from which baseline values are subtracted. Left panel values are reported as mean \pm standard deviation, whilst right panel data are presented as an individual scatter dot plot with a mean (bold horizontal lines indicate global mean changes derived from averaging all individual mean changes). $* p<0.05$ and $* * * p<0.005$ statistically significant difference when compared to baseline values, respectively
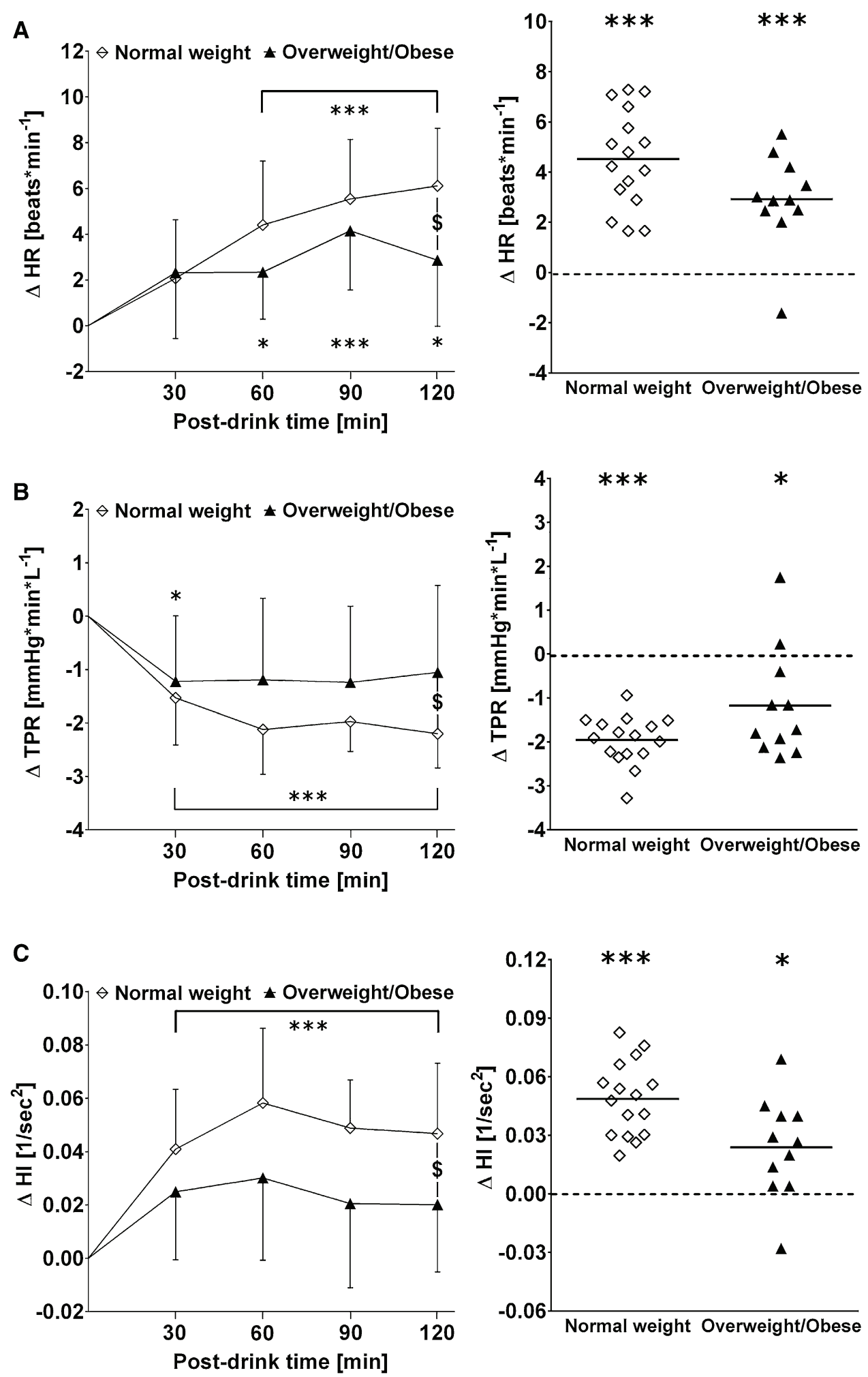

overweight/obesity than in subjects with normal weight $\left(+2.9 \pm 1.9\right.$ versus $+4.5 \pm 1.9$ beats $\mathrm{min}^{-1}$, two-way mixed model ANOVA interaction effect: $p=0.01$; Fig. 2a, left and right panel). In subjects with overweight/obesity, glucose-induced peripheral vasodilation (total peripheral resistance: $-1.2 \pm 1.3 \mathrm{vs}$. $-2.0 \pm 0.6 \mathrm{mmHg}$ min $\mathrm{L}^{-1}$, twoway mixed model ANOVA interaction effect: $p=0.04)$ and myocardial contractility (Heather index: $+0.02 \pm 0.03$ vs. 
Fig. 3 Left panels a-c Time course of changes $(\Delta)$ from baseline values over the following $30 \mathrm{~min}, 30-60 \mathrm{~min}$, 60-90 $\mathrm{min}$ and 90-120 min post-drink for stroke volume $(S V)$, cardiac output $(C O)$ and baroreflex sensitivity (BRS), respectively. Right panels represent individual mean changes in $\mathrm{SV}, \mathrm{CO}$ and BRS over 120 min post-drink, from which baseline values are subtracted. Left panel values are reported as mean \pm standard deviation, whilst right panel data are presented as an individual scatter dot plot with a mean (bold horizontal lines indicate global mean changes derived from averaging all individual mean changes.). ${ }^{*} p<0.05, * * p<0.01$, and $* * * p<0.005$ statistically significant difference when compared to baseline values, respectively
A
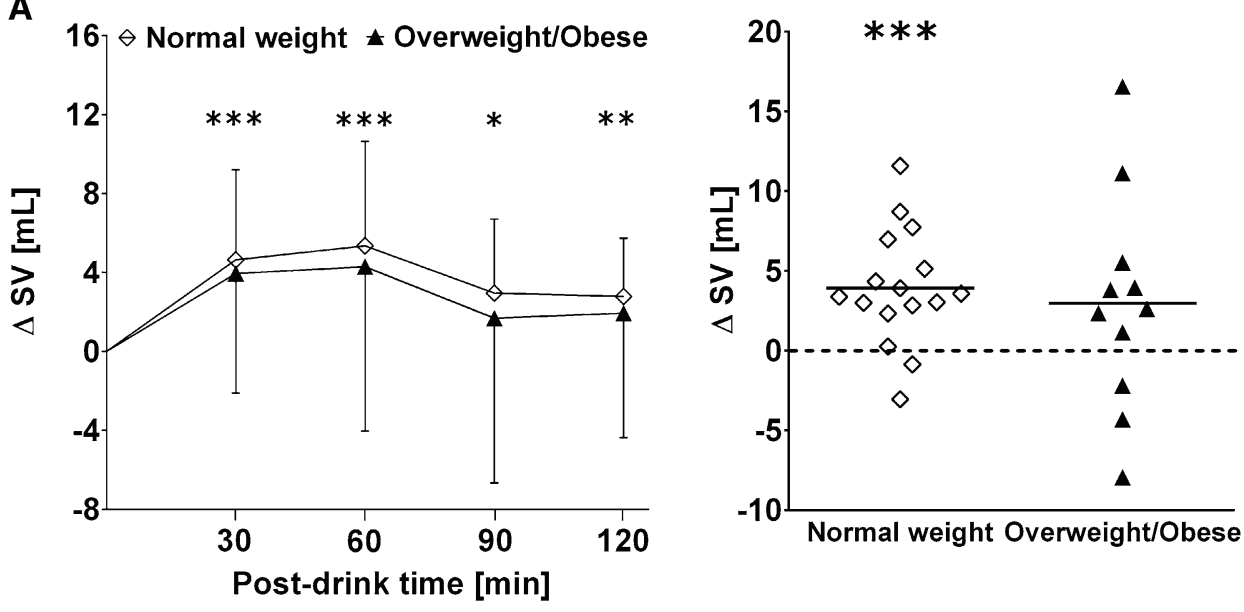

B
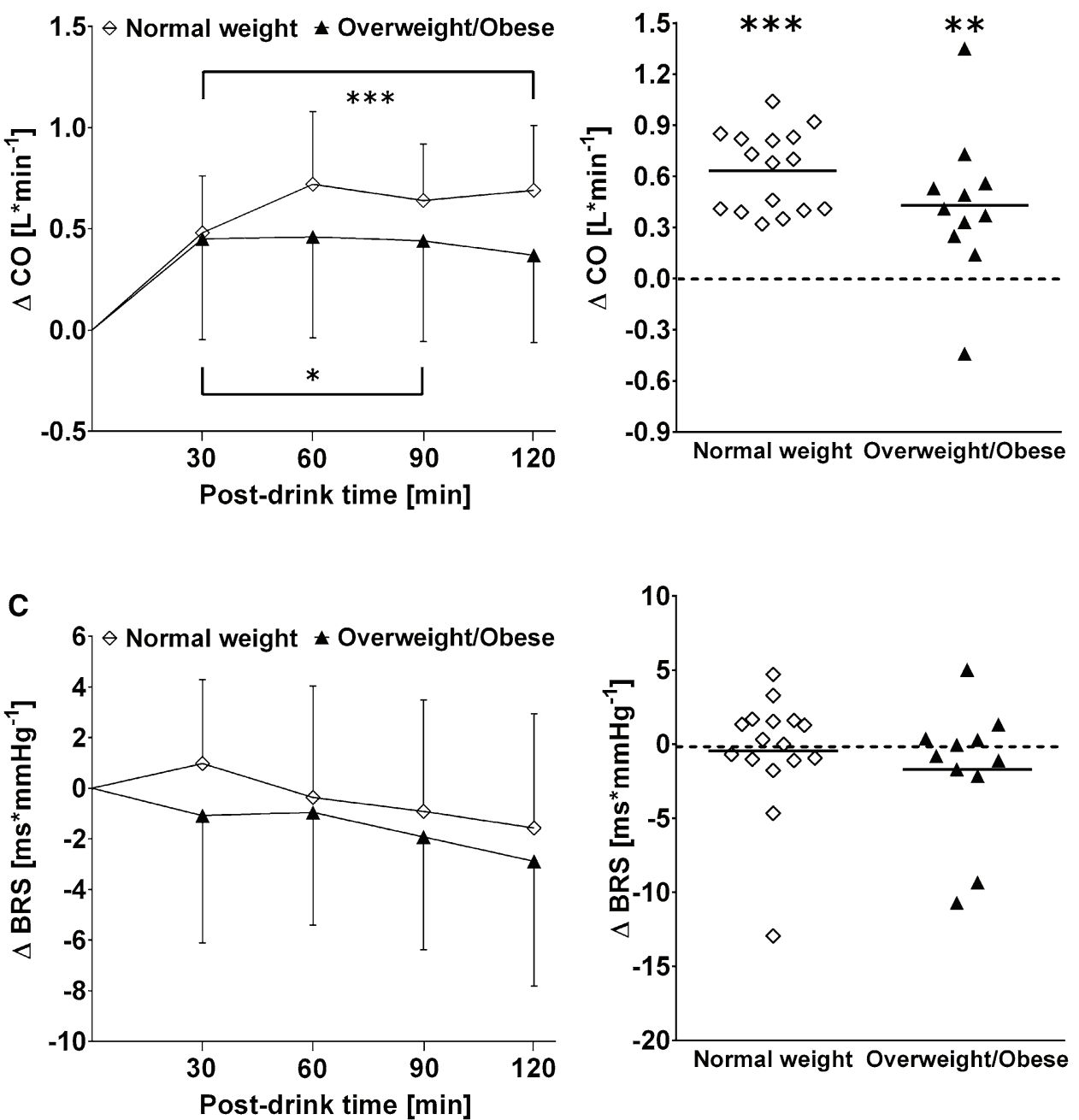

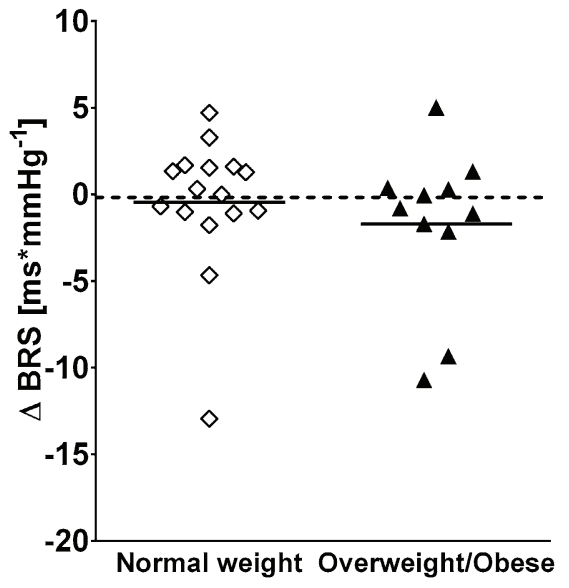

$+0.05 \pm 0.02 \mathrm{~s}^{-2}$, two-way mixed model ANOVA interaction effect: $p=0.01$ ) responses were significantly impaired compared to subjects with normal weight (Fig. 2 b and c, left and right panels).
Stroke volume, cardiac output and baroreflex sensitivity did not differ between the groups (all $p>0.10$ ) (Fig. 3, a-c left and right panels). 


\section{Glucose drink-induced changes in plasma glucose and insulin}

In response to the glucose drink, subjects with overweight/obesity showed a trend toward greater cumulative insulin response compared to the normal weight group $\left(+7445 \pm 4858\right.$ vs. $\left.4968 \pm 1924 \mathrm{mU} \mathrm{h} \mathrm{L}^{-1} ; p=0.08\right)$ (Fig. 4 b, right panel). In contrast, no significant difference was observed between the groups for plasma glucose responses to the glucose drink ( $p=0.59$, Fig. 4 a, right panel).

\section{Discussion}

We investigated in young male subjects with overweight/ obesity, but without glucose intolerance or hypertension, and age-matched male subjects with normal weight whether mild aberrations in glucose metabolism caused by a glucose drink might affect distinct regulatory control elements for blood pressure homeostasis. The present study shows that a mildly altered glucose metabolism (i.e. increased fasting glucose, insulin and HOMA-IR compared to the normal weight control group) already affects the cardiovascular system and its homeostasis by attenuating peripheral vasodilation and diminishing cardiac responses after a glucose drink. Moreover, the subjects with overweight/obesity had increased pulse pressure in response to the glucose drink, a response that was not seen in subjects with normal weight.

Glucose metabolism represents one powerful factor capable of inducing short-term perturbations in peripheral resistance that are accompanied by changes in blood flow. Indeed, seminal research shows that for individuals with normal glucose tolerance, who were in a resting state, insulin strongly stimulated the sympathetic nervous system, which emphasizes the impact of changes in plasma insulin levels on total peripheral resistance [9]. This contention was confirmed and extended by work from Baron and Brechtel [30], who showed that insulin primarily affected skeletal muscle vascular resistance and, consequently, increased cardiac output dose-dependently [30]. Moreover, and in contrast to individuals with normal weight, vascular resistance and the accompanying increase in cardiac output was blunted in obese and insulin-resistant subjects [30]. However, it is currently unknown whether subjects with overweight/obesity, but without glucose intolerance, also present signs of a perturbed vascular action of insulin in response to a glucose drink. In comparison to our control group with normal weight, we observed in male subjects with overweight/ obesity, but without glucose intolerance, a significantly impaired drop in total peripheral resistance in response to the glucose drink, which points toward a perturbation in the normal insulin-induced peripheral vasodilation. It could be
Fig. 4 Left panels $\mathbf{a}$ and $\mathbf{b}$ Time course changes in absolute values for plasma glucose and plasma insulin, respectively. Left panels represent averaged data from the respective blood-draw interval starting at baseline, denoted as 0 , and every 30 min thereafter up to 120 min. Right panels represent individual area under curve analysis using the trapezoid method for plasma glucose and plasma insulin measured over 120 min post-drink. Left panel values are reported as mean \pm standard deviation, whilst right panel data are presented as an individual scatter dot plot with a mean (bold horizontal lines indicate global mean changes derived from averaging all individual mean changes). $* p<0.05, * * p<0.01$ and $* * * p<0.005$ statistically significant difference when compared to baseline values, respectively
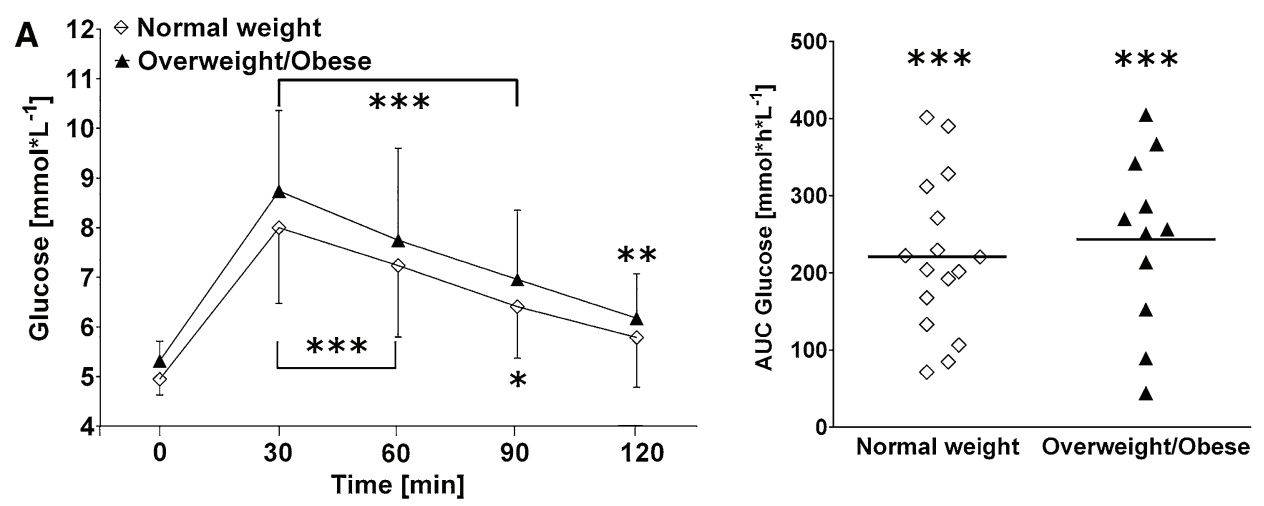

B
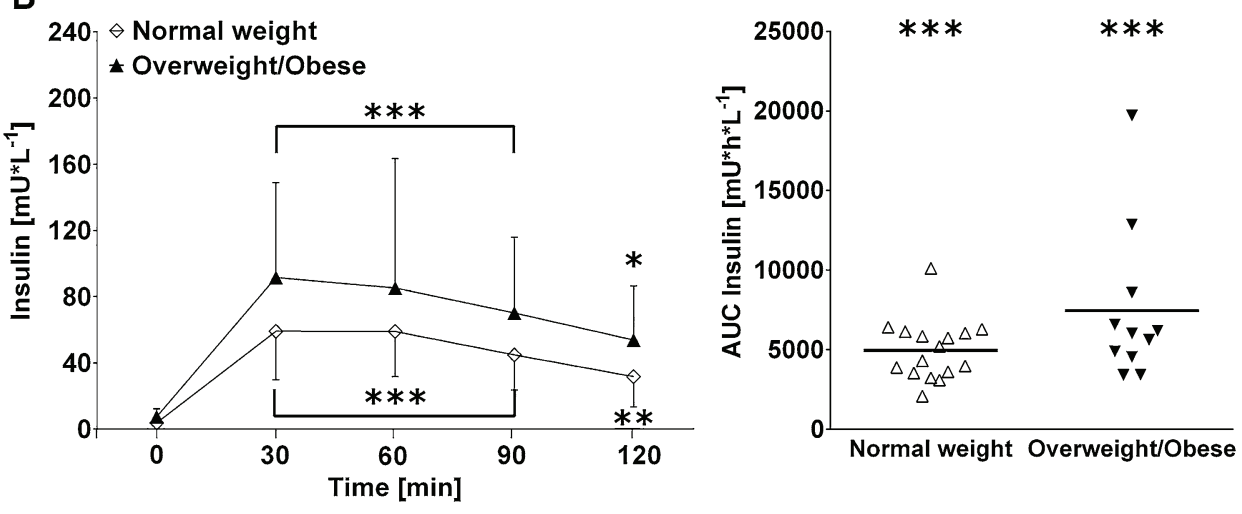
speculated that our observed acute hemodynamic effects are, at least in part, triggered by visceral intra-abdominal fat depots, which, according to the portal theory [31], adversely affect hepatic and peripheral insulin resistance. Therefore, it is suggestible that repeated and frequent intake of sugary drinks, which increases the likeliness of developing general [32] and abdominal obesity [33], could over time promote the risk of developing type 2 diabetes and cardiovascular disease. Moreover, acutely increased insulin levels are known to elevate sympathetic nervous tone [9], which, in turn, over time might adversely affect the salt sensitivity of the kidneys due to sympathetic overstimulation with the potential for blood pressure dysregulation [7].

Insulin-induced changes in cardiac output in response to the glucose drink were not observed to be different between our groups, whilst heart rate, but not stroke volume, increased in subjects with normal weight to a significantly greater level compared to subjects with overweight/obesity. Moreover, a reduced myocardial contraction force accompanied this attenuated heart rate response to the glucose drink seen in subjects with overweight/obesity. This latter finding is in agreement with a previous study, which investigated myocardial characteristics in subjects with severe obesity, overweight/obesity and normal weight that showed subclinical changes of left ventricular function even in overweight (BMI: 25-29.9 $\mathrm{kg} \mathrm{m}^{-2}$ ) and mildly obese subjects (BMI: $30-35 \mathrm{~kg} \mathrm{~m}^{-2}$ ) [34].

In response to the glucose drink, the blood pressure results from the present study demonstrate evidence toward increasing pulse pressure values in subjects with overweight/obesity, whilst systolic and diastolic blood pressure responses did not differ between groups. Although systolic blood pressure responses were not observed to be different between our groups, subjects with overweight/obesity, but not subjects with normal weight, had significantly increased systolic blood pressure over time. In agreement with our blood pressure findings stands a cross-sectional study, which found that in subjects with obesity increasing levels of body mass index were independently associated with decreasing arterial compliance, i.e. which led to increases in pulse pressure [35]. However, we were not able to discern pulse pressure differences at baseline between subjects with overweight/obesity and normal weight. Therefore, it might be that a glucose drink induced a rise in plasma insulin, which was significantly higher in subjects with overweight/obesity, and could adversely affect pulse pressure. Moreover, pulse pressure is dependent on elastic properties of Windkessel vessels and has been known to adversely affect cardiovascular outcomes [36], therefore underscoring the clinical implication of our study's finding. Another contributor toward short-term blood pressure regulation is the baroreflex where its sensitivity is suggested to be adjustable in order to match physiological adaptions to various cardiovascular perturbations [37]. In this context, we were not able to detect a difference between our groups for glucose-drink induced changes in baroreflex sensitivity, which could be potentially due to the young age of our test subjects. Indeed, La Rovere and colleagues identified age and blood pressure as the strongest predictors of baroreflex sensitivity [38], of which aging was observed to be associated with a decrease in baroreflex sensitivity [39].

Study limitations and perspective: We acknowledge that in our study the number of subjects with overweight/obesity is rather small, therefore our findings and conclusions should be considered with caution. Moreover, we only investigated a defined cohort (i.e. young male Caucasians), therefore it still needs to be determined whether our findings can be confirmed in children and adolescents, as well as in women and ethnicities other than Caucasians.

It is becoming increasingly evident that the human gut microbiota, i.e. micro-organisms that colonize the gut, might affect human metabolism [40], whilst its contribution towards the development of obesity and type 2 diabetes is currently not yet clear [41] and warrants further investigation [42]. Interestingly, non-caloric sweeteners, but not glucose or sucrose, are capable of inducing glucose intolerance by altering the gut microbiota in a mouse model, as well as in humans [43]. However, due to the low number of human subjects in this above- mentioned study $(n=7)$ [43] and the number of non-responders (three out of seven subjects showed unchanged glycaemic responses to a glucose tolerance test [43]) it remains to be established whether artificially sweetened or sugar sweetened beverages might adversely affect the gut microbiota, which would lead to dysregulation of glucose metabolism.

In conclusion, the current study sought to explore whether mild alterations in glucose metabolism, which are seen in healthy male subjects with overweight/obesity, but without glucose intolerance or hypertension, affect regulatory control elements of blood pressure homeostasis. We have chosen a standardized glucose drink in order to provoke a perturbation of blood pressure regulation and observed, in comparison to a control group with normal weight, that male subjects with overweight/obesity showed attenuated peripheral vasodilation and diminished cardiac responses associated with impaired cardiac output and myocardial contractility. Moreover, the increase in peripheral pulse pressure observed in subjects with overweight/obesity might indicate an early sign of a dysregulated blood pressure response to an oral glucose load. Finally, our results add to the growing scientific evidence that the combination of overweight/ obesity and sugar intake affects the cardiovascular system adversely and may therefore contribute to increased cardiovascular disease risk in susceptible individuals. 
Acknowledgements This work was supported by intramural funding and did not receive any grant from commercial, public, or not-for-profit agencies.

\section{Compliance with ethical standards}

Conflict of interest The authors declare no conflict of interest and no competing financial 20 interests.

\section{References}

1. Santulli G (2013) Epidemiology of cardiovascular disease in the 21th century: updated numbers and updated facts. J Cardiovasc Dis $1: 1-2$

2. Danaei G, Lu Y, Singh GM et al (2014) Cardiovascular disease, chronic kidney disease, and diabetes mortality burden of cardiometabolic risk factors from 1980 to 2010: a comparative risk assessment. Lancet Diabetes Endocrinol 2:634-647

3. Poirier P, Giles TD, Bray GA et al (2006) Obesity and cardiovascular disease: pathophysiology, evaluation, and effect of weight loss-An update of the 1997 American Heart Association Scientific Statement on obesity and heart disease from the Obesity Committee of the Council on Nutrition, Physical Activity, and Metabolism. Circulation 113:898-918

4. Klein S, Burke LE, Bray GA et al (2004) Clinical implications of obesity with specific focus on cardiovascular disease: a statement for professionals from the American Heart Association Council on Nutrition, Physical Activity, and Metabolism: endorsed by the American College of Cardiology Foundation. Circulation 110:2952-2967

5. Kahn SE, Hull RL, Utzschneider KM (2006) Mechanisms linking obesity to insulin resistance and type 2 diabetes. Nature 444:840-846

6. Fox CS, Golden SH, Anderson C et al (2015) Update on prevention of cardiovascular disease in adults with type 2 diabetes mellitus in light of recent evidence. Circulation 132(8):691-718

7. Landsberg L, Aronne LJ, Beilin LJ et al (2013) Obesity-related hypertension: pathogenesis, cardiovascular risk, and treatment: a position paper of The Obesity Society and the American Society of Hypertension. J Clin Hypertens (Greenwich) 15(1):14-33

8. Monnard CR, Grasser EK (2018) Perspective: cardiovascular responses to sugar-sweetened beverages in humans: a narrative review with potential hemodynamic mechanisms. Adv Nutr 9:70-77

9. Rowe JW, Young JB, Minaker KL, Stevens AL, Pallotta J, Landsberg L (1981) Effect of insulin and glucose infusions on sympathetic nervous system activity in normal man. Diabetes 30:219-225

10. Ferrannini E, Natali A, Bell P, Cavallo-Perin P, Lalic N, Mingrone G (1997) Insulin resistance and hypersecretion in obesity. J Clin Invest 100:1166-1173

11. Monnard CR, Fellay B, Scerri I, Grasser EK (2017) Substantial inter-subject variability in blood pressure responses to glucose in a healthy, non-obese population. Front Physiol 8:507

12. Angeli F, Reboldi G, Trapasso M (2019) European and US guidelines for arterial hypertension: similarities and differences. Eur J Intern Med 63:3-8

13. World Health Organization, World Health Organization (2006) Definition and diagnosis of diabetes mellitus and intermediate hyperglycemia: report of a WHO/IDF consultation. World Health Organization, Geneva, pp 1-50

14. American Diabetes Association (2014) Diagnosis and classification of diabetes mellitus. Diabetes Care 37:S81-S90
15. Wilson TD, Reinhard DA, Westgate EC et al (2014) Social psychology. Just think: the challenges of the disengaged mind. Science 345:75-77

16. Monnard CR, Grasser EK (2017) Water ingestion decreases cardiac workload time-dependent in healthy adults with no effect of gender. Sci Rep 7:7939

17. Jeleazcov C, Krajinovic L, Münster T et al (2010) Precision and accuracy of a new device (CNAPTM) for continuous noninvasive arterial pressure monitoring: assessment during general anaesthesia. Br J Anaesth 105(3):264-272

18. Ilies C, Bauer M, Berg P et al (2012) Investigation of the agreement of a continuous non-invasive arterial pressure device in comparison with invasive radial artery measurement. Br J Anaesth 108(2):202-210

19. Hill DW, Merrifield AJ (1976) Left ventricular ejection and the Heather Index measured by non-invasive methods during postural changes in man. Acta Anaesthesiol Scand 20:313-320

20. Cybulski G (2011) Ambulatory impedance cardiography. The systems and their application. Lecture Notes in Electrical Engineering, 76. Springer-Verlag, Berlin

21. Kubicek WG, Patterson RP, Witsoe DA (1970) Impedance cardiography as a noninvasive method of monitoring cardiac function and other parameters of cardiovascular system. Ann N Y Acad Sci 170:724-732

22. Fortin J, Habenbacher W, Heller A et al (2006) Non-invasive beat-to-beat cardiac output monitoring by an improved method of transthoracic bioimpedance measurement. Comput Biol Med 36:1185-1203

23. Bertinieri G, di Rienzo M, Cavallazzi A, Ferrari AU, Pedotti A, Mancia G (1985) A new approach to analysis of the arterial baroreflex. J Hypertens Suppl 3(3):S79-S81

24. Hunma S, Ramuth H, Miles-Chan JL et al (2016) Body composition-derived BMI cut-offs for overweight and obesity in Indians and Creoles of Mauritius: comparison with Caucasians. Int J Obes (Lond) 40:1906-1914

25. Browning LM, Mugridge O, Chatfield MD et al (2010) Validity of a new abdominal bioelectrical impedance device to measure abdominal and visceral fat: comparison with MRI. Obesity (Silver Spring) 18:2385-2391

26. Matthews DR, Hosker JP, Rudenski AS, Naylor BA, Treacher DF, Turner RC (1985) Homeostasis model assessment: insulin resistance and beta-cell function from fasting plasma glucose and insulin concentrations in man. Diabetologia 28:412-419

27. Chinali M, de Simone G, Roman MJ et al (2006) Impact of obesity on cardiac geometry and function in a population of adolescents: the Strong Heart Study. J Am Coll Cardiol 47:2267-2273

28. Garavaglia GE, Messerli FH, Nunez BD, Schmieder RE, Grossman E (1988) Myocardial contractility and left ventricular function in obese patients with essential hypertension. Am J Cardiol 62:594-597

29. Abel ED, Litwin SE, Sweeney G (2008) Cardiac remodeling in obesity. Physiol Rev 88:389-419

30. Baron AD, Brechtel G (1993) Insulin differentially regulates systemic and skeletal muscle vascular resistance. Am J Physiol 265:E61-E67

31. Item F, Konrad D (2012) Visceral fat and metabolic inflammation: the portal theory revisited. Obes Rev 13:30-39

32. Dhingra R, Sullivan L, Jacques PF et al (2007) Soft drink consumption and risk of developing cardiometabolic risk factors and the metabolic syndrome in middle-aged adults in the community. Circulation 116(5):480-488

33. Anari R, Amani R, Veissi M (2017) Sugar-sweetened beverages consumption is associated with abdominal obesity risk in diabetic patients. Diabetes Metab Syndr 11:S675-S678

34. Wong CY, O'Moore-Sullivan T, Leano R, Byrne N, Beller E, Marwick TH (2004) Alterations of left ventricular 
myocardial characteristics associated with obesity. Circulation 110:3081-3087

35. Kwagyan J, Tabe CE, Xu S, Maqbool AR, Gordeuk VR, Randall OS (2005) The impact of body mass index on pulse pressure in obesity. J Hypertens 23:619-624

36. Payne RA, Wilkinson IB, Webb DJ (2010) Arterial stiffness and hypertension. Emerging concepts. Hypertension 55(1):9-14

37. Taylor CE, Witter T, El Sayed K, Hissen SL, Johnson AW, Macefield VG (2015) Relationship between spontaneous sympathetic baroreflex sensitivity and cardiac baroreflex sensitivity in healthy young individuals. Physiol Rep 3(11):e12536

38. La Rovere MT, Pinna GD, Raczak G (2008) Baroreflex sensitivity: measurement and clinical implications. Ann Noninvasive Electrocardiol 13(2):191-207
39. Kardos A, Watterich G, de Menezes R, Csanády M, Casadei B, Rudas L (2001) Determinants of spontaneous baroreflex sensitivity in a healthy working population. Hypertension 37(3):911-916

40. Gérard C, Vidal H (2019) Impact of gut microbiota on host glycemic control. Front Endocrinol (Lausanne) 30(10):29

41. Harsch IA, Konturek PC (2018) The role of gut microbiota in obesity and type 2 and type 1 diabetes mellitus: new insights into "old" diseases. Med Sci 6(2):pii: E32

42. Menaa F (2015) Are probiotics pro-obesity or potential anti-obesity agents? J Food Nutr Sci 2(1):1

43. Suez J, Korem T, Zeevi D et al (2014) Artificial sweeteners induce glucose intolerance by altering the gut microbiota. Nature 514(7521):181-186 